\title{
DIFICULDADES NO DIAGNÓSTICO CLÍNICO E ELETRENCEFALOGRÁFICO DE LIPOFUSCINOSE CERÓIDE NEURONAL
}

\author{
Cleurecy Oliveira Vasques' ${ }^{1}$, Rosa Maria Figueiredo Valério², \\ Umbertina Conti Reed ${ }^{3}$, Rosi Mary Grossman ${ }^{4}$, Fernando Kok ${ }^{5}$
}

\begin{abstract}
RESUMO - Tradicionalmente, as lipofuscinoses ceróides neuronais (LCN) eram classificadas de acordo com a idade de início e características clínicas em quatro grandes grupos. Recentemente, os estudos genéticos possibilitaram uma classificação mais pormenorizada dessa entidade em oito formas, permitindo o diagnóstico mais preciso de casos previamente considerados atípicos. Por outro lado, foi demonstrado que mutações de um mesmo gene poderiam ser responsáveis por grande variedade de fenótipos clínicos. O objetivo deste estudo é apresentar dois irmãos com achados clínicos e eletrencefalográficos compatíveis com a forma juvenil de LCN mas com alterações ultra-estruturais características da forma infantil tardia dessa doença. Os achados eletrencefalográficos auxiliam no diagnóstico da LCN, mas pouco contribuem na sua classificação.
\end{abstract}

PALAVRAS-CHAVE: lipofuscinose ceróide neuronal, eletrencefalograma, microscopia eletrônica, fenótipo, genótipo.

\begin{abstract}
Pitfalls in the clinical and electroencephalographic diagnosis of ceroid lipofuscinosis
ABSTRACT - Neuronal ceroid lipofuscinosis ( $\mathrm{NCL}$ ) were traditionally classified according to age of onset and clinical features in four main groups. Recently, a combination of clinical, ultra structural and genetics data led to the recognition of eight forms of NCL, providing a more precise framework to classify atypical cases. By the other hand, it was shown that mutations in the same gene could be responsible for a large variety of clinical phenotypes. The objective of this study is to describe two brothers with clinical and electroencephalographic abnormalities characteristic of juvenile NCL, but with ultra structural abnormalities suggestive of late infantile NCL. Electroencephalogram is useful for clinical diagnosis of NCL but it is not helpful in its classification.
\end{abstract}

KEY WORDS: neuronal ceroid lipofuscinosis, electroencephalogram, electron microscopy, phenotype, genotype.

As lipofuscinoses ceróides neuronais constituem grupo geneticamente heterogêneo de doenças neurodegenerativas caracterizadas clínicamente por deterioração cognitiva, epilepsia mioclônica e perda visual progressiva. Têm herança autossômica recessiva e ocorrem com mais frequência na infância e adolescência, tendo incidência estimada de 0,1 a 7,0 para 100000 nascidos vivos ${ }^{1,2}$. O termo lipofuscinose ceróide neuronal (LCN) reconhece o achado neuropatológico mais característico: neurônios degenerados com acúmulo de lipofuscina, um pigmento ainda não caracterizado quimicamente de maneira adequada ${ }^{1,2}$.

A associação entre idade de início e achados ultraestruturais possibilitou o reconhecimento de quatro formas de LCN: 1. LCN infantil ou doença de
Haltia-Santavuori, com início entre 6 meses e 2 anos de idade e caracterizada pela presença na pele ou conjuntiva de depósitos osmiofílicos granulares; 2 . LCN infantil tardia ou doença de Jansky-Bielschowsky, de início entre início 2 e 4 anos e com presença de corpos curvilíneos; 3. LCN juvenil ou doença de Spielmeyer-Vogt-Sjögren, de início entre 4 e 10 anos de idade e com inclusões citoplasmáticas similares a impressões digitais. 4. Forma adulta de $L C N$ ou doença de Kufs, com início entre 15 e 50 anos e com padrão ultrastrutural heterogêneo, com presença de corpos curvilíneos e inclusões digitiformes, associados ou não a depósitos osmiofílicos granulares ${ }^{2-5}$.

Graças ao avanço da genética molecular tornouse possível classificar as LCNs em oito, e não apenas quatro formas, e identificar seis dos genes associa-

Serviço de Eletrencefalografia e Serviço de Neurologia Infantil da Divisão de Clínica Neurológica do Hospital das Clínicas da Faculdade de Medicina da Universidade de São Paulo, São Paulo SP, Brasil (FMUSP): 'Médica Estagiária do Serviço de Eletrencefalografia; ${ }^{2}$ Doutora e Médica Assistente do Serviço de Eletrencefalografia; ${ }^{3}$ Livre Docente e Chefe do Serviço de Neurologia Infantil; ${ }^{4}$ Doutora e Chefe do Serviço de Eletrencefalografia; ${ }^{5}$ Doutor e Médico Assistente do Serviço de Neurologia Infantil

Recebido 16 Março 2004, recebido na forma final 29 Julho 2004. Aceito 23 Setembro 2004.

Dra. Cleurecy Oliveira Vasques - Departamento de Neurologia do HCFMUSP - Avenida Dr Enéias de Carvalho Aguiar, 255/5andar - 05403-000 São Paulo SP - Brasil. E-mail: cleurecy@uol.com.br 
dos com LCN: CLN1, CLN2, CLN3, CLN5, CLN6 e CLN8. O genes CLN7 já foi mapeado porém não identificado e o gene responsável pela forma adulta de LCN (CLN4) não foi até o momento mapeado ou identificado'. As oito formas conhecidas até o momento de LCN são as seguintes:

CLN1, responsável pela totalidade dos casos infantis da LCN, decorrente de deficiência da enzima lissômica proteína palmitoil tioesterase (PPT). Em todos os pacientes até o momento estudados encontrou-se presença à microscopia eletrônica (ME) de depósitos osmiofílicos granulares. Sabe-se hoje que a idade de início dessa forma de LCN é mais variável do que se imaginou inicialmente, sendo descritas formas de início neonatal, infantil precoce e tardia, juvenil ou na vida adulta. Os sintomas clínicos iniciais variam com a idade de início. São conhecidos mais de 30 mutações do gene CLN1. O diagnóstico pode ser feito por meio da determinação da atividade da PPT em leucócitos e confirmado por meio da análise molecular do gene CLN1 ${ }^{1}$.

CLN2, responsável pela maior parte dos casos de LCN infantil tardia clássica, cujos primeiros sintomas aparecem entre 2 e 4 anos, decorre de deficiência da enzima lisossomal tripeptidil-peptidase 1 (TPP1). Usualmente esta forma se inicia com epilepsia, sendo a mioclonia o tipo de crise característico. Involução do desenvolvimento neuropsicomotor é evidenciada precocemente, seguida por demência, ataxia e sinais piramidais e extrapiramidais. O comprometimento visual aparece entre $4 \mathrm{e}$ 6 anos de idade, podendo o eletrorretinograma ser normal no início e logo se tornar extinto. $\mathrm{O}$ achado de corpos curvilíneos à ME facilita o diagnóstico, mas alguns pacientes apresentam outros tipos de inclusões, como digitiformes ou complexos retilineares. Atualmente reconhece-se maior diversidade fenotípica na LCN por mutação de CLN2, sendo a idade de início apenas um dos elementos sugestivos. O diagnóstico pode ser realizado pela análise da atividade TPP1 e confirmado pela análise mutacional do gene CLN2. Sabe-se que outros genes, entre os quais CLN1, CLN5, CLN6 e CLN7 podem resultar em fenótipos similares, porém com níveis normais de tripeptidilpeptidase $1^{1}$.

CLN3, responsável pela maior parte dos casos de LCN juvenil, é decorrente de mutação que interfere com a função da proteína battenina, de função desconhecida e que se localiza na membrana lisossomial. Os sintomas iniciais incluem comprometimento visual rápido e progressivo, seguido por distúrbios da fala, declínio lento das funções cognitivas e epilepsia. Inclusões digitiformes características dessa forma de LCN são encontradas à ME de linfócitos ou em material de biópsia e autópsia. Até o momento, nenhum marcador bioquímico permite o diagnóstico de LCN juvenil mas uma única mutação, conhecida como deleção de $1 \mathrm{~Kb}$, é responsável por mais de $70 \%$ das mutações responsáveis por CLN3 ${ }^{1}$.

CLN4 é o nome reservado para a forma adulta da LCN. No entanto, o gene para essa condição até o momento não foi localizado e discute-se se essa forma é decorrente da mutação em um ou mais dos genes atualmente reconhecidos de LCN. O diagnóstico é baseado somente em informações clínicopatológicas. Os sinais e sintomas iniciais usualmente aparecem em torno dos 30 anos de idade e incluem epilepsia mioclônica de difícil controle, demência, ataxia e sinais piramidais e extrapiramidais tardios, geralmente sem comprometimento visual. A demonstração de inclusões características das LCN em material de biópsia é essencial para o diagnóstico definitivo, podendo ser encontrada mistura de inclusões (digitiformes, corpos curvilíneos e depósitos osmiofílicos granulares) ${ }^{1}$.

CLN5, responsável pela variante finlandesa ou forma infantil tardia da LCN. As diferenças principais entre a LCN infantil tardia clássica e a variante finlandesa são o início mais tardio da doença, usualmente 4 a 7 anos, e a presença de complexos retilineares e inclusões digitiformes à $\mathrm{ME}^{1}$.

CLN6, responsável pela variante cigana/hindu da forma infantil tardia da LCN. Comprometimento visual e crises epilépticas podem aparecer no início da doença. Quando o início ocorre após os 4 anos, os sintomas incluem ataxia. A ME demonstra mistura de inclusões (inclusões digitiformes, corpos curvilìneos, complexos retilineares) ${ }^{1}$.

CLN7, responsável pela variante turca da forma infantil tardia da LCN. O início ocorre entre 1 e 6 anos e a maioria das famílias é consangüínea. O fenótipo é similar ao da CLN2 clássica, mas difere na idade de início. A ME mostra inclusões digitiformes ou mistura de inclusões (inclusões digitiformes, corpos curvilíneos, complexos retilineares) ${ }^{1}$.

CLN8, responsável pela epilepsia progressiva com retardo mental. $O$ início dos sintomas ocorre entre 5 e 10 anos, sendo o diagnóstico baseado no quadro clínico (epilepsia, retardo mental e disfunção motora). A ME mostra depósito de material que lembra corpos curvilíneos e depósitos osmiofílicos granulares ${ }^{1}$.

O objetivo deste estudo é revisar na literatura, os achados clínicos e padrões eletrencéfalográficos de pacientes com diagnóstico de LCN confirmado por exames neurofisiológicos e ultraestrutural, correlacionando-os aos estágios evolutivos da doença e de apresentar o caso de dois irmãos, que demonstram as dificuldades encontradas na classificação da LCN 


\section{CASOS}

Caso 1 - Paciente com 15 anos, primeiro filho de uma prole de três. Os períodos gestacional, peri e pós $n$ a $t$ a I cursaram sem qualquer intercorrência. Nascido de parto normal com peso de $3700 \mathrm{~g}$. A investigação familiar demonstrou que várias gerações das famílias materna e paterna são provenientes da mesma cidade do interior da Bahia e, portanto, não pode ser afastada anscestralidade comum. Há referência, ainda, de três primos paternos com perda da acuidade visual na infância e epilepsia, sem diagnóstico definido. Evoluiu com desenvolvimento neuropsicomotor adequado até os 6 anos de idade quando começou a apresentar perda da acuidade visual e fotofobia, com piora rápida e progressiva. Aos 7 anos de idade estava amaurótico.

Aos 8 anos foi avaliado pela primeira vez e o exame neurológico demonstrava distúrbio do comportamento e rebaixamento cognitivo, marcha instável e amaurose. Evoluiu com piora do distúrbio comportamental, cognitivo e motor. O eletrencefalograma (EEG) obtido em sono e a tomografia de crânio (TC) foram normais. Três meses após, começou a apresentar crises epilépticas do tipo mioclônico que foram facilmente controladas com ácido valpróico. Um ano após, já com controle medicamentoso das crises epilépticas, o EEG obtido em vigília demonstrava ritmo dominante posterior a $7 \mathrm{~Hz}$, pouco reativo à abertura e fechamento ocular, além de atividade epileptiforme de projeção nas regiões temporal média esquerda e parietal esquerda e mediana, independentes, não sendo verificadas anormalidades à fotostimulação intermitente em baixas freqüências.

Atualmente o quadro clínico se mantém estável e as crises epilépticas controladas.

Caso 2 - Paciente com 11 anos de idade, irmão mais novo do propósito, com antecedentes gestacionais, peri e pós natais negativos e desenvolvimento neuropsicomotor adequado até os 5 anos de idade. Aos 4,5 anos iniciou perda progressiva da acuidade visual, além de dor ocular e lacrimejamento. Apresentava, ainda, agitação e irritabilidade que pioravam durante processos infecciosos.

Aos 6 anos foi avaliado pela primeira vez apresentando ao exame neurológico apenas alteração de fundo de olho, que demonstrava papilas pálidas e acinzentadas, além de escassez de vasos. O desenvolvimento neuropsicomotor encontrava-se adequado para a idade do paciente. O eletrorretinograma mostrou-se extinto e a TC foi normal. Foi submetido a EEG em vigília, que mostrou ritmo dominante posterior discretamente alentecido, com predomínio de ondas a $7 \mathrm{~Hz}$, pouco reativo a abertura e fechamento ocular, além de paroxismos de ondas agudas de projeção na região parietal direita. Não foram observadas anormalidades à fotostimulação intermitente em baixas freqüências. A biópsia da conjuntiva demonstrou corpos curvilíneos.

Atualmente o paciente se mantém sem crises epilépticas, porém com intenso distúrbio do comportamento e involução cognitiva.

\section{DISCUSSÃO}

O quadro clínico dos dois irmãos iniciou-se com perda rápida e progressiva da acuidade visual com cerca de 6 anos de idade e evoluiu com declínio cognitivo e distúrbio comportamental; apenas no caso 1 foram encontrados sinais motores piramidais e epilepsia mioclônica, que teve início após dois anos de evolução da doença e foi facilmente controlada. As características clínicas desses dois pacientes são compatíveis com a encontrada na forma juvenil da LCN, cujos sinais e sintomas clínicos usualmente aparecem entre 4 e 7 anos, com comprometimento visual rápido e progressivo ${ }^{3,4,6}$. Nessa forma de LCN também evidencia-se degeneração pigmentar da retina e atrofia do nervo óptico ${ }^{7}$; distúrbios da fala e declínio lento das funções cognitivas ocorrendo após os 8 a 14 anos de idade ${ }^{3,4,8}$; epilepsia aparecendo dos 5 aos 18 anos, mais comumente após os 12 anos $^{8-10}$; problemas de comportamento, sinais extrapiramidais e distúrbios do sono completando o quadro clínico na segunda década de vida ${ }^{4,11}$. O eletrorretinograma e potencial evocado visual são precocemente anormais ${ }^{12,13}$ e os exames de imagem revelam atrofia cerebral e, em menor grau, atrofia cerebelar após os 15 anos $^{1,2,14}$.

Nos pacientes aqui relatados, apesar do quadro clínico ser superponível ao encontrado na forma juvenil da LCN, o estudo anátomo-patológico demonstrou presença de corpos curvilíneos, o que é característico de CLN2 ${ }^{1,15,16}$. Sabe-se atualmente que indivíduos com mutação em CLN2 podem tanto ter o fenótipo característico da forma infantil tardia como da juvenil ${ }^{1,4,17}$. Desta forma, o estudo a natomo-patológico é importante para o direcionamento correto da investigação.

Nos nossos pacientes, os EEGs obtidos no primeiro ano de evolução da doença apresentavam alentecimento do ritmo dominante posterior, com pouca reatividade à abertura e fechamento ocular. Este achado é concordante com os da literatura, apesar de grande parte das casuísticas incluírem pacientes com a forma infantil ou infantil tardia de LCN. Alguns autores referem que a diminuição da reatividade do ritmo dominante posterior à abertura e fechamento ocular é a anormalidade neurofisiológica mais precoce dessa doença- ${ }^{7-9,12}$. É relatado também 
o desaparecimento precoce dos elementos próprios do sono, especialmente dos fusos ${ }^{7,11,13,14}$. Apenas no caso 1 foi obtido EEG durante o sono, que se apresentou normal. Em ambos os casos não foram observadas anormalidades à fotostimulação intermitente, mesmo em freqüências baixas. A presença de espículas posteriores, desencadeadas pela fotostimulação intermitente em freqüência baixa, é um achado importante e prevalente nas formas infantil e infantil tardia de LCN27,18,19.

$O$ achado de anormalidades eletrencefalográficas pouco específicas em fases precoces da LCN, como encontrado nos nossos pacientes, não afasta o diagnóstico da doença. $O$ conhecimento da existência desses padrões, assim como a comparação com exames repetidos em intervalos curtos, é importante para adequada correlação clínico - eletrencefalográfica. Existem poucas descrições quanto aos padrões eletrencefalográficos da forma juvenil de LCN. Sainio ${ }^{13}$ refere que não existem características específicas no EEG da forma juvenil da LCN, podendo ser normal nos estágios precoces da doença e evoluir com alentecimento da atividade de base, além de aparecimento de atividade epileptiforme aos 8-9 anos de idade ${ }^{7,10,13}$.

A caracterização da atividade epileptiforme nas diferentes formas de LCN também é escassa na literatura, uma vez que as casuísticas maiores incluem apenas pacientes com as formas infantil e infantil tardia. Veneselli et al. ${ }^{2}$ descreveram 3 padrões de EEG em vigília: a - paroxismos de espículas e ondas agudas de alta voltagem se repetindo em intervalos de 8-10 segundos, nas fases mais precoces da doença ou de 2-4 segundos, nas fases mais tardias, adquirindo características pseudoperiódicas; b - atividade epileptiforme contínua, constituída por espículas e ondas lentas; c - alterações paroxísticas multifocais. O padrão pseudoperiódico se apresenta mais bem definido em vigília do que durante sono. Outros autores também relataram a presença de atividade epileptiforme em surtos ou de maneira contínua ${ }^{2,13}$, de projeção focal, multifocal ou generalizada ${ }^{13,19-21}$.

Nossos pacientes apresentaram EEG com paroxismos epileptiformes focais, concordando com Veneselli et al. ${ }^{2}$ que referiram presença de surtos de espículas e ondas lentas, encontrados freqüentemente em pacientes com a forma juvenil de LCN. Vanhanen et al. ${ }^{7}$ referiram alentecimento e ulterior atenuação da amplitude, evoluindo para silêncio elétrico cerebral nas fases tardias da doença, podendo haver sobrevida de vários anos após o achado de EEG isoelétrico. Essas alterações provavel- mente refletem a destruição rápida e progressiva do córtex cerebral em pacientes com LCN 16,19.

Em resumo, o diagnóstico da $L C N$ depende de uma combinação de dados clínicos, bioquímicos, ultra-estruturais e moleculares, e apenas os elementos clínicos não permitem uma correta classificação dessa condição. O EEG é um elemento de suporte ao diagnóstico e tampouco contribui para a sua classificação.

Agradecimento - Ao Professor Sérgio Rosemberg pela sua importante colaboração.

\section{REFERÊNCIAS}

1. Wisniewski KE, Zhong N, Philippart M. Pheno/genotypic correlations of neuronal ceroid lipofuscinoses. Neurology 2001;57:576-581.

2. Veneselli E, Biancheri R, Buoni S, Fois A. Clinical and EEG findings in 18 cases of late infantile neuronal ceroid lipofuscinosis. Brain Dev 2001; 23:306-311.

3. Santavuori P. Neuronal ceroid-lipofuscinoses in childhood. Brain Dev 1988;10:80-83.

4. Berkovic SF, Cochius J, Andermann E, Andermann F. Pro g ressive myoclonus epilepsies: clinical and genetics aspects. Epilepsia 1993; 34(Suppl):S19-S30.

5. Santavuori P, Haltia M, Rapola J. Infantile type of so-called neuronal ceroid-lipofuscinosis. Dev Med Child Neurol 1974;16:644-653.

6. Lauronen L, Munroe PB, Järvelä I, et al. Delayed classic and protracted phenotypes of compound heterozygous juvenile neuronal ceroid lipofuscinosis. Neurology 1999;52:360-365.

7. Vanhanen SL, Sainio K, Lappi M, Santavuori P. EEG and evoked potentials in infantile neuronal ceroid-lipofuscinosis. Dev Med Child Neurol 1997;39:456-463.

8. Acharya JN, Satishchandra P, Shankar SK. Familial progressive mioclonus epilepsy: clinical and eletrophysiologic observations. Epilepsia 1995;36:429-434.

9. AicardiJ, Plouin P, Goutiéres F. Ceroid-lipofuscinoses. Rev EEG Neurophysiol 1978;8:149-159.

10. Elligson RJ, Schain RJ. EEG patterns in juvenile cerebral lipidosis. Electroenceph Clin Neurophysiol 1969;27:191-194.

11. Kirveskari E, Partinen M, Salmi T, et al. Sleep alterations in juvenile neuronal ceroid-lipofuscinosis. Pediatr Neurol 2000;22:347-354.

12. Pampiglione G, Harden A. So-called neuronal ceroid lipofuscinosis neurophysiological studies in 60 chidren. J Neurol Neurosurg Psychiatry 1977;40:323-330.

13. Sainio K. Neurophysiological findings in neuronal ceroid lipofuscinoses. Neuropediatrics 1997;28:70.

14. Santavuori P, Raininko R, Vanhanen S-L, Launes J, Sainio K. MRI of the brain, EEG sleep spindles and SPECT in the early diagnosis of infantile neuronal ceroid lipofuscinosis. Dev Med Child Neurol 1992;34:61-79.

15. Santavuori P, Haltia M, Rapola J, Raitta C. Infantile type of so-called ne u ronal ceroid-lipofuscinosis: a clinical study of 15 patients. J Neurol Sci 1973;18:257-267.

16. Haltia M, Rapola J, Santavuori P, Keränen A. Infantile type of so-called neuronal ceroid-lipofuscinosis: morphological and biochemical studies. J Neurol Sci 1973;18:269-285.

17. Wisniewski KE, Zhong N, KaczmarskiW, et al. Compound heterozygous genotype is associated with protracted juvenile neuronal ceroid lipofuscinosis. Ann Neurol 1998;43:106-110.

18. NaqviSZ, Beach RL, Armao DM, Greenwood RS. Photoparoxysmal response in late infantile neuronal ceroid-lipofuscinosis. Pediatr Neurol 1998;19:395-398.

19. Santavuori P. EEG in the infantile type of so-called neuronal ceroid-lipofuscinosis. Neuropädiatrie 1973;4:375-387.

20. Santavuori P, Westermarck T, Rapola J, et al. Antioxidant treatment in Spielmeyer-Sjögren's disease. Acta Neurol Scand 1985;71:136-145.

21. Percy AK. The inherited neurodegenerative disorders of childhood: clinical assessment. J Child Neurol 1987;2:82-97. 